\title{
Propuesta de conversión de fincas convencionales a fincas agroecoturísticas para el desarrollo sustentable del Cantón Machala
}

\author{
Conversion proposal of conventional farms to \\ agroecotouristic farms to sustainable development \\ of Machala Canton
}

Nugra Betancourth, Mónica Alexandra

Universidad Técnica de Machala

(Machala - Ecuador)

manugra@utmachala.edu.ec

Calle Iñiguez, Melissa Universidad Técnica de Machala (Machala - Ecuador) mpcalle@utmachala.edu.ec

Ortiz Berrezueta, Lilia Argentina Universidad Técnica de Machala

(Machala - Ecuador) lortiz@umet.edu.ec

Palomeque Jaramillo, Jean Michel Universidad Técnica de Machala (Machala - Ecuador) jmpalomeque@utmet.edu.ec

Revista Cumbres Vol.5 №2

Versión impresa ISSN 1390-9541

Versión electrónica ISSN 1390-3365

http://investigacion.utmachala.edu.ec/revistas/index.php/Cumbres 


\title{
RESUMEN
}

La investigación tiene como finalidad buscar alternativas viables para el desarrollo del agroturismo y la ecología en nuestro país, aprovechando bondades geográficas y climáticas para la generación del turismo durante todo el año. Machala en los últimos diez años se ha ido convirtiendo en un polo de desarrollo económico y social, dando lugar a que empresas multinacionales asienten sus negocios contribuyendo al desarrollo turístico, partiendo del problema central ¿Que provoca el bajo desarrollo agroturistico productivo en Machala? Llegando a plantear el objetivo general: Elaborar una propuesta de conversión de fincas convencionales a fincas agroecoturisticas para el desarrollo del turismo del cantón Machala. Se realizó una investigación de tipo descriptiva y correlacionar, no experimental, además de ser exploratoria. Al aplicar las encuestas las unidades investigativas compuestas por productores agrícolas del cantón, se pudo conocer su interés por llevar a cabo la investigación, tomando en cuenta la obtención de beneficios económicos adicionales permitiendo a los turistas puedan observar e interactuar en las diferentes actividades agrícolas que se dan, se busca promocionar que las fincas convencionales tomen agroecológicas para un producto sustentable, y culturalizar a la comunidad la forma de compaginar dos actividades como la agricultura con la turística, obteniendo ingresos adicionales.

Palabras clave: Agroturismo, ecología, fincas agroecoturísticas, actividades agrícolas.

\begin{abstract}
The present research has as purpose to find viable alternatives for the development of agrotourism and ecology in our country taking advantage of the geographical and climatic benefits for the generation of tourism throughout the year. Machala in the last ten years has become a pole of economic and social development, giving rise to multinational companies settle their businesses contributing to tourism development, starting from the central problem $\dot{ }$ What causes the low productive agroecotouristic development in Machala? Coming to state the general objective: to elaborate a conversion proposal of conventional farms to agroecotouristic farms to develop Machala Canton tourism. A descriptive and correlating research was realized, not experimental, besides being exploratory. When applying the surveys the research units composed of agricultural producers of the Canton, it was possible to know their interest to carry out the research, taking into account obtaining additional economic benefits allowing tourists to observe and interact in the different agricultural activities that happen, it seeks to promote that conventional farms take agroecological for a sustainable product, and
\end{abstract}


culturalize the community how to combine two activities such as agriculture with tourism, obtaining additional incomes.

Keywords: Agrotourism, ecology, agroecotouristic farms, agricultural activities.

\section{INTRODUCCIÓN}

En la actualidad las nuevas tendencias del mercado a nivel internacional han convertido al turismo en principal movimiento que permite el desarrollo sostenible de los pueblos, marcando una perspectiva de optimización en el aprovechamiento de los recursos culturales vinculados al medio natural, el Ecuador se considera entre los diecisiete países megadiversos del mundo, tiene grandes recursos naturales, pero también ha sufrido un gran impacto de las actividades productivas (Silva, 2015). La mayor ventaja comparativa con la que cuenta el país es su biodiversidad, por ello es fundamental saberla aprovechar de manera adecuada, mediante su conservación y su uso sustentable.

La agricultura es uno de los ejes principales sobre los que se desenvuelve la economía del país. Al ser esta una actividad fundamental tanto en el ámbito económico como en la seguridad alimentaria, se vuelve crucial conocer su evolución a lo largo de los años, con el objetivo de observar el comportamiento de la producción y su sostenibilidad en el tiempo. (Monteros Guerrero, Sumba Lusero, \& Salvador Sarauz, 2014).

El turismo rural es unánimemente aceptado y unitario utilizado para una u otra de las áreas de recepción de los turistas, respectivamente, realizando servicios turísticos. (Ş. \& Apetri, 2008). El recurso natural nos proporciona la naturaleza, sin alteración por parte del ser humano. Los recursos turísticos se basan en los potenciales geográficos, históricos monumentales, culturales, a hechos sociales que satisfacen las necesidades de la demanda (Navarro, 2015).

La oferta turística es el conjunto de bienes y servicios turísticos que los productores del sector están dispuestos a ofrecer a determinados precios, son demandados por los turistas y complementan el producto turístico final (Bateman, Harris, \& Leisure, 2011). La calidad ambiental representa, por definición, las características cualitativas y/o cuantitativas inherentes al ambiente en general o medio particular, de los ecosistemas de un sistema de indicadores de sostenibilidad (Luque, 2009).

Se debe aprovechar la presencia de fincas y haciendas en las cercanías de la ciudad de Machala con la intención de diseñar modelos para fincas agroecoturísticas productivas que fomenten el desarrollo turístico del cantón, conocer sus costumbres, conocimientos ancestrales y su diario vivir, demostrando al turista como es el funcionamiento del sector bananero, cacaotero, con lo que se busca desarrollar una propuesta de calidad ajustada a la realidad socioeconómica de los productores agrícolas del cantón Machala, creando a su vez fuentes de trabajo que van de la mano con el cambio de la matriz productiva, y del buen vivir que promueve el actual gobierno. 
En "México y en Latinoamérica, la diversificación que esta alcanzado la actividad turística en el medio rural, desemboca en dos conceptos integradores: ecoagroturismo y agroecoturismo social. En el caso del Ecuador, en el año 2003 el Ministerio de Turismo "solicitó a la Organización Mundial de Turismo (OMT) una Misión para definir el Programa de Turismo Rural en el Ecuador. Llegando a plantearnos el siguiente problema:¿Cuáles serán los beneficios o efectos de transformar en fincas agroecoturísticas las unidades de producción convencionales localizadas en el Cantón Machala, provincia de El Oro? El trabajo investigativo reviste de originalidad, debido a que no se ha encontrado trabajos similares, situación que sin duda favorecerá a los intereses teóricos de la investigadora.

Por tal motivo el objetivo general será Elaborar una propuesta de conversión de fincas convencionales en fincas agroecoturísticas para el desarrollo del turismo del cantón Machala, provincia de El Oro, para lo que tendrá que realizar un diagnóstico situacional del agroecoturismo productivo en las fincas agrícolas del cantón Machala de la provincia de El Oro, proponer lineamientos y estrategias para el desarrollo de fincas agroecoturísticas del cantón Machala, provincia de El Oro y elaborar un plan de marketing para la finca agroecoturística del cantón Machala, provincia de El Oro.

\section{MATERIALES Y MÉTODOS}

\section{Modalidad y tipo de investigación}

El tipo de investigación fue descriptiva y correlacional por cuanto se analizan situaciones, será no experimental por cuanto no se manipularán las variables, teniendo la intención de observar el fenómeno tal como se da en su contexto, para luego analizarlos, pudiendo sacar conclusiones que ayuden a la investigación así mismo fue exploratoria debido a que recaba información no reconocida sobre un fenómeno o problema, dando un acercamiento a los productores agrícolas y su posibilidad de implantar el agroecoturismo como una alternativa para su economía.

\section{Métodos}

Los aspectos metodológicos aplicados fueron de un estudio cuantitativo de carácter descriptivo, donde se utilizaron los métodos: inductivo, deductivo, histórico lógico, sistémico estructural y modelación.

\section{Variables}

\section{Tipo de variable}

La variable independiente de la presente investigación es: Modelo de finca agroecoturística productiva.

El modelo de finca agroecoturística productiva es un esquema que se deberá de seguir para transformar una finca convencional en ecoturística. 


\section{Categoría}

La categoría es nominal.

\section{Indicador}

Los indicadores Capacitación del personal, Insumos, Equipos, Maquinaria, Capital de trabajo, Financiamiento

\section{Tipo de variable}

La variable dependiente de la presente investigación es:

Desarrollo turístico sostenible

\section{Población y Muestra}

El universo para nuestro caso de estudio estará integrado por los productores agrícolas pequeños y medianos localizados en el cantón Machala. El universo está compuesto por 3358 personas dedicadas a la siembra de banano, cacao, ganado porcino, avícolas. Como el universo es amplio, será necesario aplicar una muestra, necesitándose aplicar la siguiente fórmula:

$$
\begin{aligned}
& \mathrm{Tm}=\frac{\mathrm{N}}{1+(\Sigma \mathrm{A})^{2} \times \mathrm{N}} \\
& \mathrm{Tm}=\frac{3.358}{1+(0.05)^{2} \times 3.358} \\
& \mathrm{Tm}=\frac{3.358}{9,395} \\
& \mathrm{Tm}=357.42 \\
& \mathrm{Tm}=357
\end{aligned}
$$

Al aplicar la fórmula se obtiene que el tamaño de muestra (n) corresponde a 357 productores agrícolas que tienen sus fincas en la ciudad de Machala.

Modelo de finca agroecoturística En nuestro país existen lugares turísticos que permiten que el turista esté en contacto con la naturaleza, además de tener acceso a fincas donde puede compenetrarse con el proceso productivo generado por los agricultores de estos sectores. Un ejemplo es la Cabaña del Río, localizada en Puerto Quito a 140 kilómetros de la capital de la República del Ecuador, Quito, al noroccidente de la provincia de Pichincha. Uno de los servicios desplegados por esta empresa es la visita a fincas de campo que quedan a su alrededor, donde se "podrá realizar tours guiados y tener acceso a probar una variedad de frutas exóticas, desconocidas en el resto del mundo. Se encontrarán frutas para la exportación donde el turista tendrá la oportunidad de degustarlas" (Forest-Rain, 2008). 


\section{RESULTADOS}

En la investigación de campo se pudo determinar la ausencia de diagnósticos situacionales del agroecoturismo productivo en las fincas del cantón Machala, por lo que se procedió a su realización, cuyos resultados se los presenta a continuación:

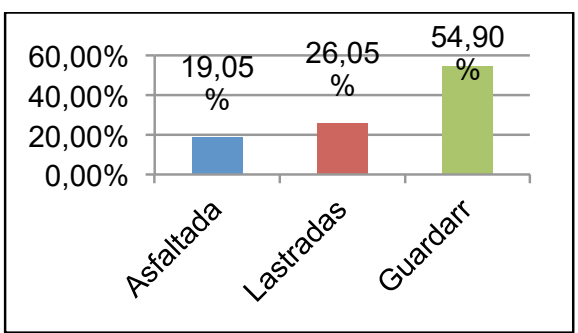

Figura 1. Estado de vías de acceso, la mayoría de fincas poseen vías de acceso de segundo orden.

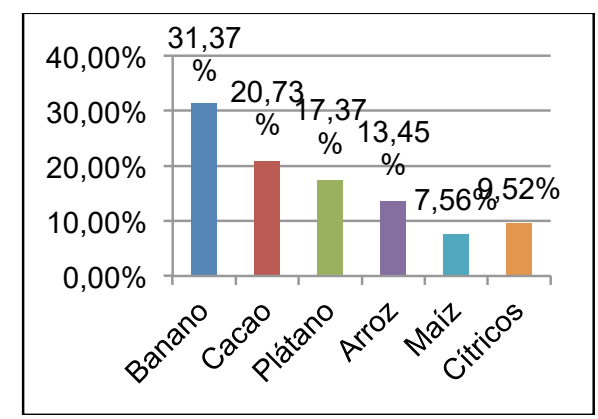

Figura 3. Los Tipo de plantación agrícola con un $31,37 \%$ son las de banano

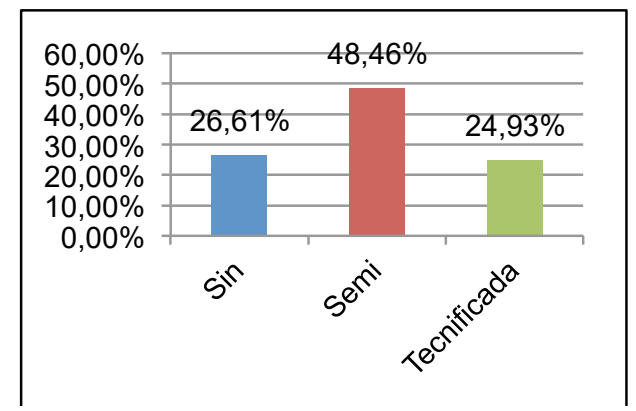

Figura 5. Nivel de tecnificación, la mayoría de fincas no son tecnificadas, por lo que sus propietarios podrían ver con interés la presente propuesta como mecanismo para incrementar sus ingresos.

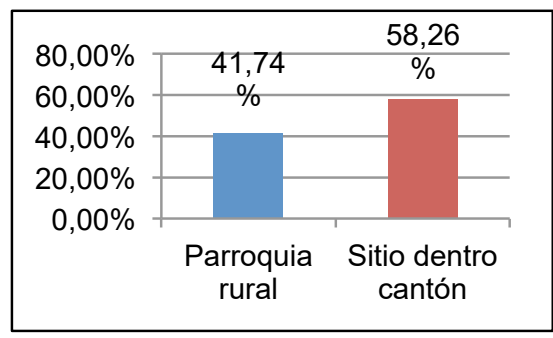

Figura 2. Localización de la finca agrícola un $58,26 \%$ se encuentran en la zona rural del Cantón Machala como El Cambio y el Retiro

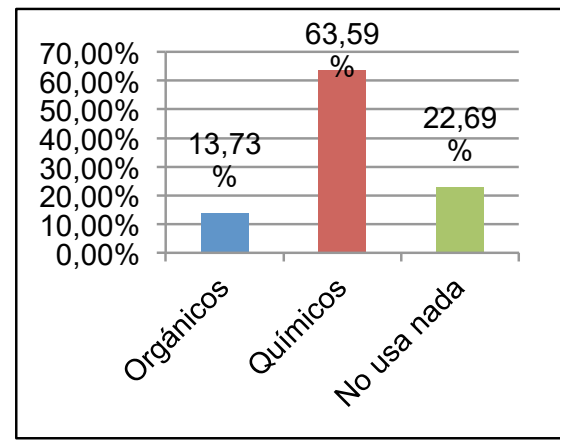

Figura 4. Utilización de insumos agrícolas, la mayoría de fincas no son orgánicas, situación que requiere del apoyo de los agricultores, así como una inversión para su conversión.

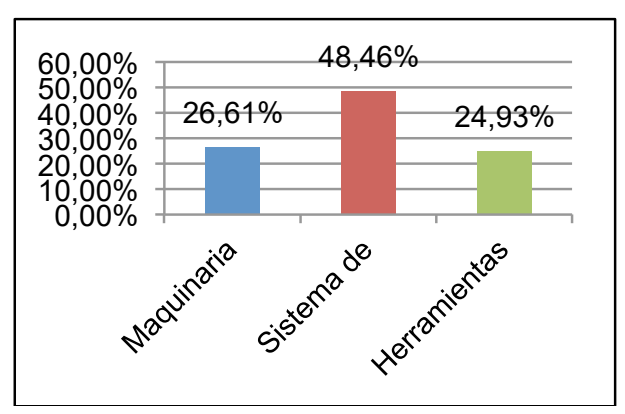

Figura 6. Equipos con que cuenta la finca, se evidencia que dependiendo del tamaño de la finca los productores cuentas con maquinarias, utensilios o sistemas de riego para sus actividades diarias. 


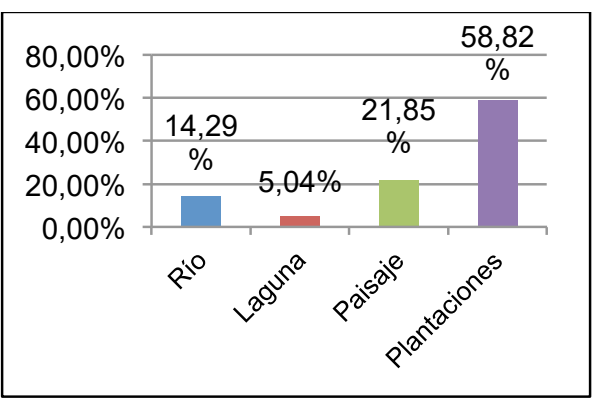

Figura 7. Atractivo turístico de la finca agrícola, serían sus plantaciones agrícolas, sus paisajes y algunas se encuentran cerca de ríos y lagunas.

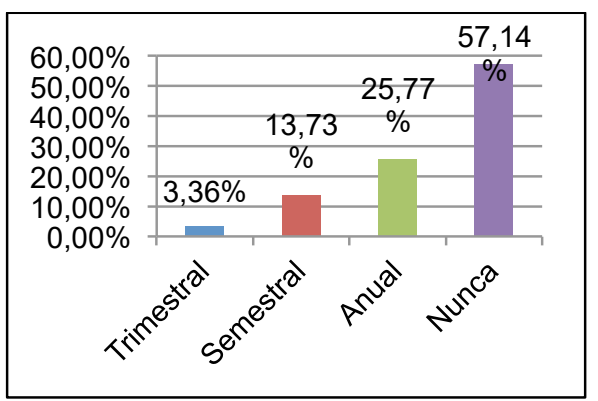

Figura 9. Capacitación del personal, consideran que es un gasto y no una inversión, en perjuicio de sus intereses económicos y productivos.

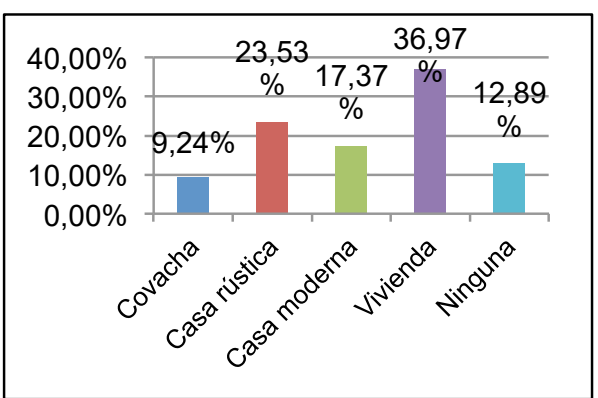

Figura 8. Tipo de construcción en finca agrícola, la mayoría posee viviendas en su finca de diferentes tipos.

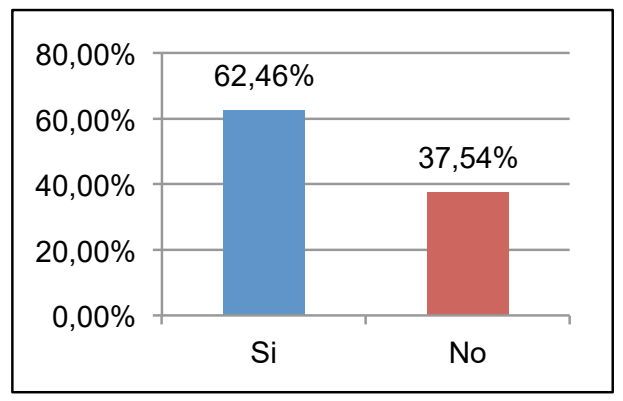

Figura 10. Interés de convertir a finca agroecoturística, en su mayoría con un $62,46 \%$ los productores agrícolas señalaron que si estarían interesados, por lo se puede observar un alto grado de interés, necesitándose de una clara exposición del tema, convirtiendo al agroecoturismo como una oportunidad para incrementar su economía.

Con esta modalidad la comunidad tendría más opciones de ingresos económicos como lo menciona (Guibert, Grosso, Arbeletche, \& Bellini, 2011) ofreciendo conocimiento de ese patrimonio y al mismo tiempo la adquisición de los productos para su consumo; teniendo en cuenta la época de siembra y el inicio y el período de las cosechas (Espeitx, 2004) para que ellos puedan apreciar de mejor manera las plantaciones que se hacen en la zona, fincas representativas de las diferentes condiciones de paisaje presentes en la zona piloto (Salmón, Funes-Monzote, \& Martín, 2012).

Así mismo realizar adecuaciones, ya que son la imagen del lugar y el turista debe llevarse una buena impresión de lo que visita y sobre todo sentirse cómodo y seguro; por tal motivo (Blanco \& Riveros, 2010) dice que, es necesario adecuar las instalaciones de modo que los visitantes puedan efectuar recorridos por el lugar y observar e incluso participar en los procesos, cuando las condiciones lo permitan.

Por tal motivo, (Martínez, Pauls, \& Solsona, 2003) señala que, las pernoctaciones son generadas en el periodo de máxima afluencia de turistas, por el cual el ingreso económico para los prestadores de servicios turísticos podría aumentar en el desarrollo de turismo del cantón. 


\section{Lineamientos y estrategias para el desarrollo de fincas agroecoturísticas del cantón Machala}

El modelo para el desarrollo de fincas agroecoturísticas en la ciudad de Machala estará conformado operacionalmente por cinco fases las cuales se describen a continuación:

Tabla 1. Fases del modelo para el desarrollo de fincas agroturísticas

\begin{tabular}{|l|l|l|}
\hline ACTIVIDAD & DESCRIPCIÓN & DURACIÓN \\
\hline Fase I & $\begin{array}{l}\text { Promoción del proyecto a productores agrícolas con el apoyo de } \\
\text { organismos competentes. }\end{array}$ & 2 meses \\
\hline Fase II & Mejora de la infraestructura turística & 2 meses \\
\hline Fase III & Educación ambiental para productores agrícolas y turistas & \\
\hline & 2 meses & \\
\hline Fase IV & $\begin{array}{l}\text { Capacitación a productores agrícolas y empleados de las fincas } \\
\text { agroecoturísticas }\end{array}$ & 2 meses \\
\hline Fase V & Promoción turística & 2 meses \\
\hline
\end{tabular}

\section{Plan de marketing para las fincas agroecoturísticas del cantón Machala}

El análisis PEST se refiere al análisis de factores externos que podrían afectar a la finca agroecoturísticos estos factores son: Políticos, Económicos, Sociales y Tecnológicos del contexto. El análisis PORTER permite conocer el nivel de competencia que existe en un determinado mercado el poder de negociación de los compradores, de los proveedores, amenaza de nuevos competidores entrantes, amenaza de productos sustitutos, rivalidad entre los competidores y el mercado objetivo.

\section{CONCLUSIONES}

Realizando el diagnostico situacional del agroecoturismo productivo podemos señalar que el Cantón Machala es el centro productivo y económico de la provincia de El Oro, su mayor producción agrícola es el banano, cacao y plátano; la mayoría de las fincas están en la zona rural y no son orgánicas, poseen diferentes tipos de viviendas, el principal atractivo turístico serían sus plantaciones y algunas poseen ríos, lagunas; existe interés de parte de los agricultores en convertir la finca convencional a agroecoturistica, necesitándose una clara explicación mediante capacitaciones y esto son nuevas oportunidades de mejorar la economía y su calidad de vida, además dará a conocer al Ecuador y al mundo sus tradiciones y forma de vida.

Al desarrollar proyectos agro turísticos con tecnología ecológica en una finca muy cerca de la ciudad de Machala, permitirá que sus habitantes tengan un espacio donde compartir vivencias de la vida campestre.

Los lineamientos y estrategias para el desarrollo de fincas agroecoturisticas del Cantón Machala estará conformado operacionalmente por cin- 
co fases con una duración de dos meses cada una y son de promoción del proyecto a productores con el apoyo de organismos competentes, mejora de infraestructura turística, educación ambiental para productores y turistas, capacitación para productores, empleados agrícolas y promoción turística.

Al desarrollar el plan de marketing para la finca agroecutirstica del cantón Machala, se realizó el análisis PEST y el análisis PORTER y se determinó el logo, slogan, estrategias publicitarias en prensa escrita, redes sociales, folletos, eventos y ferias turísticas, creando un impacto positivo, posicionando al agroecoturismo en una forma sustentable y sostenible de hacer turismo, creando una concienciación positiva en los habitantes del cantón Machala.

\section{REFERENCIAS BIBLIOGRÁFICAS}

Bateman, H., Harris, E. y., \& Leisure, K. D. (2011). Travel and Tourism. Concepcion: Mira Rueda.

Blanco, M., \& Riveros, H. (2010). El agroturismo como diversificación de la actividad agropecuaria y agroindustrial. Análisis, 21-29.

da Cruz, G., Velozo, T., \& Falcao, A. (2011). Twitter, Youtube e innovación en la promoción turística online: Análisis de las estrategias del Ministerio de Turismo de Brasil. Estudios y perspectivas en Turismo, 627-642.

Espeitx, E. (2004). Patrimonio alimentario y turismo: una relación singular. PASOS. Revista de Turismo y Patrimonio Cultural, 193-213.

Guibert, M., Grosso, S., Arbeletche, P., \& Bellini, M. E. (2011). De Argentina a Uruguay: espacios y actores en una nueva lógica de producción agrícola. PAMPA, 13-38.

López-Roldán, P., \& Fachelli, S. (2016). La Encuesta. Metodología de la investigación social cuantitativa. Universitat Autonoma de Barcelona , 1-41. Obtenido de https://ddd.uab.cat/pub/caplli/2016/163567/metinvsoccua_a2016_ cap2-3.pdf

Luque, A. F. (27 de Marzo de 2009). EL TURISMO RURAL EN ESPAÑA: TERMINOLOGÍA Y PROBLEMAS. ENTRECULTURAS, I(1), 8-17.

Martínez, F., Pauls, A., \& Solsona, J. (2003). LAS VIVIENDAS FAMILIARES Y SU USO TURÍSTICO EN LA COMUNIDAD VALENCIANA. Estudios Turísticos, 159-177.

Monteros Guerrero, A., Sumba Lusero, E., \& Salvador Sarauz, S. (2014). PRODUCTIVIDAD AGRÍCOLA EN EL ECUADOR. Quito: Dirección de Análisis y Procesamiento de la Información, Coordinación General del Sistema de Información Nacional Ministerio de Agricultura, Ganadería, Acuacultura y Pesca. Obtenido de http://www.redalyc.org/html/904/90431109054/

Navarro, D. (2015). Recursos turísticos y atractivos turísticos: CONCEPTUALIZACIÓN, clasificación y valoració. Cuadernos de Turismo(10.6018/turismo.35.221641), 335-357.

Pulido Polo, M. (2015). Ceremonial y protocolo: métodos y técnicas de investigación científica. Opción , 31(1), 1137-1156. Obtenido de http://www. redalyc.org/pdf/310/31043005061.pdf 
S., C. I., \& Apetri, N. (2008). DEVELOPING AGROTURISM IN ROMANIA A CHALLENGE OF THE UE INTEGRATION. Suceava: University "Stefan cel Mare". Obtenido de file://C:/Users/Acer/Downloads/1075-4131-1-PB.pdf

Salmón, Y., Funes-Monzote, F., \& Martín, O. (2012). Evaluación de los componentes de la biodiversidad en la finca agroecológica "Las Palmitas" del municipio Las Tunas. Pastos y Forrajes, 321-332.

Silva, W. (2015). La problemática ambiental y el deterioro de los recursos naturales en el Ecuador. Una perspectiva desde la geografía. (Bachelor's thesis, PUCE), 1-116. Obtenido de http://repositorio.puce.edu.ec/handle/22000/866

\section{Cumbres}

